\title{
ENTRE LO ACCIDENTAL Y LO APARENTE: LA PECULIAR CONSTELACIÓN CAUSAL DEL AZAR SEGÚN ARISTÓTELES
}

\author{
Gabriela Rossi \\ Pontificia Universidad Católica de Chile \\ grossi@uc.cl
}

\begin{abstract}
This paper deals with Aristotle's concept of chance, such as it is presented in Physics II 4-6. The central section of the article concentrates on an analysis of Aristotle's definition of chance and its essential peculiarities: the fact of being an incidental (efficient) cause and the fact of existing in the domain of what is for the sake of an end. According to Rossi, both characteristics would correspond to a causal aspect (in an incidental sense) and to a non causal aspect (or just apparently causal) of chance. Finally, the author also tries to show the structural connection between the aforementioned aspects, taking as a key point the thesis of coincidence among the formal, final, and efficient causes.

Key words: Aristotle, Physics, chance, causes.
\end{abstract}

\section{Resumen}

Este artículo trata el concepto aristotélico de azar, tal como se lo presenta en Física II 4-6. La sección central del artículo se concentra en el análisis de la definición aristotélica de azar y sus peculiaridades esenciales: el hecho de ser una causa accidental (eficiente) y el hecho de existir en el dominio de lo que es en vista

\footnotetext{
${ }^{*}$ Recibido: 24-11-05. Aceptado: 19-03-06.

*Este trabajo fue realizado en el marco de una Beca Doctoral de CONICYT, Chile. Deseo expresar aquí mi inmenso agradecimiento y reconocimiento a mi actual Director de Tesis, el Prof. Dr. Alejandro Vigo, con quien mi deuda es difícil de medir, pues todos los temas aquí tratados han alcanzado su maduración actual gracias a sus innumerables y sabios aportes y a su generosa dedicación a lo largo de múltiples coloquios y reuniones individuales. No quiero implicar con ello que él comparta todas las tesis que aquí expongo, ni que sea responsable, mucho menos, de los errores que yo pueda aún haber deslizado.
} 
de un fin. Según Rossi, ambas características corresponderían a un aspecto causal (en un sentido accidental) y a un aspecto no causal (o sólo aparentemente causal) del azar. Por último, la autora también trata de mostrar la conexión estructural entre los aspectos antes mencionados, tomando como hecho clave la tesis de la coincidencia entre las causas formal, final y eficiente.

Palabras clave: Aristóteles, Física, azar, causas.

\section{Introducción}

En apenas tres breves capítulos de su Física, Aristóteles trata específicamente y expone por única vez de modo sistemático lo que puede considerarse su teoría del azar. Los capítulos 4 al 6 del libro II de la Fís. contienen, incluso, la primera reflexión filosófica sistemática de que tenemos noticia sobre este asunto, al menos en la Grecia antigua, pues si bien anteriores filósofos, como los atomistas e incluso Platón, habían echado mano ya de este concepto, especialmente para dar cuenta de procesos de tipo cosmológico e incluso cosmogónico, no obstante no es posible encontrar en ellos una reflexión temática sobre el concepto de azar, ni mucho menos sobre su naturaleza específica en cuanto causa. Aristóteles acomete por primera vez esta empresa teórica, y lo hace, paradójicamente (como es su costumbre), para despojar este concepto de una suerte de esclerosamiento hipostático adquirido en el curso de una evolución que, partiendo probablemente de los usos cotidianos, lo había depositado en las alturas cosmogónicas, y devolverlo, en cambio, a su ámbito prefilosófico originario y más familiar: el práctico, y además —a partir de él—al natural sublunar. De hecho, el fenómeno del azar, anunciándose por medio de repentinas e imprevistas irrupciones de lo fáctico e inmanejable en nuestros planes (sea para frustrar nuestras acciones o para secundarlas), resulta una experiencia básica del actuar humano, no menos ni más en el mundo antiguo que en nuestros días. Esto a tal punto que la mera expectativa de su posibilidad y la advertencia del riesgo que ella conlleva se perciben frecuentemente como un componente esencial de la condición humana.

La problemática del azar entra en la escena de la filosofía teórica que tiene por objeto los entes sujetos a movimiento inmediatamente a continuación de que se han examinado los cuatro modos en que puede 
entenderse "causa" ( $\alpha$ ítıov), y de los que deberá hacerse cargo el filósofo de la naturaleza al estudiarla científicamente. Este hecho no es él mismo en absoluto azaroso, puesto que, como ya sabemos, sea a partir de las opiniones comunes, sea a partir de ciertas tesis filosóficas anteriores, la

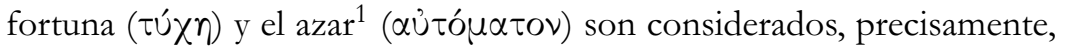
como causas de muchas cosas (cf. Fís. II 4, 195b31-36) ${ }^{2}$. Aristóteles, sobre este punto de partida, intentará mostrar cuáles son los criterios que subyacen a esta atribución causal (es decir, los criterios que permiten identificar un evento como producto del azar) tomándola tal como se presenta fundamentalmente en la concepción prefilosófica, pero también procurará exponer cuál es el sustento ontológico de tal tipo de fenómeno. Podría decirse, en este orden de cosas, que los capítulos de Fís. II 4-6 explicarían, al mismo tiempo, por qué el azar no ha sido incluido en la doctrina de las cuatro causas como una más entre ellas, ${ }^{3}$ y de qué modo puede a su vez subsumirse bajo alguna de ellas; pues, claro está, si es posible dar cuenta del sentido en que el azar es una causa a partir del marco conceptual que ofrece la teoría de las causas presentada en Fís. II

\footnotetext{
${ }^{1}$ No me ocuparé en este trabajo puntualmente de la diferencia entre estos dos conceptos, y por ello utilizaré en la mayor parte del escrito el término "azar" para referirme

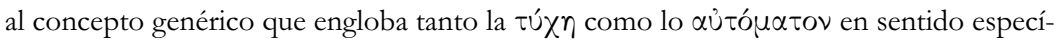
fico (cf. 197a36-b1).

${ }^{2}$ Simplicio apunta en relación a esto que filósofos como Empédocles, Demócrito y Anaxágoras, si bien no tematizaron el azar explícitamente como una causa, se sirvieron del mismo en sus explicaciones (cf. In Phys., 327.16-26), que algunos poetas, por su parte, atribuyen casi todo a la fortuna como causa (Ib. 327.27-28 y s.) y que la mayoría de la gente (oi $\pi 0 \lambda \lambda \circ i$ ) le atribuye todo (Ib. 328.5-6). Con respecto al uso filosófico que tiende a atribuirle al azar un papel en la cosmogonía y a negárselo en el ámbito práctico y natural sublunar (Fís. 196a1-7, 196a24-35; Part. Anim. I 1, 641b15-23; cf. Met. I 3, 984b11-18), Aristóteles es tajante en cuanto a la falsedad de dicha tesis (cf. 196a35-b5).

${ }^{3}$ Esto no hubiera sido tan descabellado como podría parecer en primera instancia si se considera, como decía más arriba, que en algunas concepciones filosóficas anteriores, el azar constituía una de las causas; tal es el caso por ejemplo de los atomistas (cf. DK 67A11, 68A66, A69, A70, B118), quienes además lo consideraban como idéntico con la necesidad (cf. DK 68A1, A66, A83), e incluso para Platón, que lo considera como una

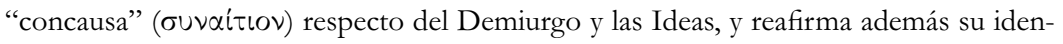
tidad con la necesidad (cf. Timeo, esp. 46c-48a, 56c, 69b). En Leyes X, 888e ss., puede encontrarse también, en el marco de la discusión del ateísmo, una exposición sincrética de las teorías presocráticas que postulaban al azar como causa a nivel cosmogónico.
} 
3, se hace con ello razonable el no haberlo contado en ese capítulo como una causa por sí al mismo nivel que las otras cuatro ${ }^{4}$. La estrategia explicativa de Aristóteles respecto del azar sería entonces, en cierto modo, reduccionista; aunque esto no implica por sí mismo, no obstante, que él conciba al azar como un fenómeno carente de consistencia ontológica (por ejemplo, al modo de una causa meramente imaginaria, o como una expresión cosmética de la propia ignorancia de las causas de un evento).

Así, la primera cuestión que propone investigar Aristóteles al comienzo de Fís. II 4 es cómo encajan la fortuna y el azar en el esquema de las causas mencionadas antes (195b33-34), esquema que involucra no sólo los cuatro modos de entender "causa" (i.e., como forma, como materia, como aquello de donde proviene el principio primero del movimiento, y como aquello 'con vistas a lo cual'), sino también los modos de enunciar o presentar cada una de estas causas en una proposición causal explicativa. En este punto me interesa concentrarme en este trabajo, es decir, en el peculiar estatus que tendría el azar como causa según la concepción de Aristóteles, y su relación, por lo tanto, con los diferentes tipos de causa presentados en II 3. En vistas de ello dejaré de lado los problemas que atañen a la distinción introducida en Fís. II 6 entre fortuna y azar como sendos tipos de lo azaroso en sentido amplio, los cuales se dan respectivamente en el ámbito de la praxis y por fuera del mismo ${ }^{5}$. En cambio, me concentraré más bien en las dos notas, tan fundamentales como difíciles de compatibilizar, que involucra la caracterización del azar en sentido genérico que realiza Aristóteles en Fís. II 5. Me refiero

\footnotetext{
${ }^{4}$ Es importante, en efecto, recordar que los cuatro modos en que puede entenderse "causa" son todos ellos por sí; cf. Fís. $195 \mathrm{a} 5$.

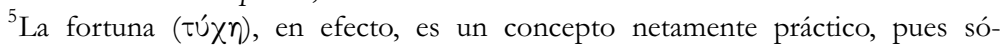
lo puede darse en y para aquellos agentes que son capaces de elección deliberada

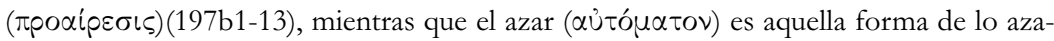
roso que se da por fuera de dicho ámbito, esto es: (i) en los agentes que no son capaces de decisión deliberada (197b14-32) y (ii) en el plano estrictamente natural (sublunar), fundamentalmente en las generaciones naturales espontáneas, cf. 197b32-35; Met. VII 7, 1032a28-32; Gen. Anim. esp. III 11; Ross (1936: 524, ad 197b32-37). Que la fortuna, en su sentido específico, cae bajo el ámbito de estudio de la filosofía práctica, resulta evidente también por el lugar que otorga Aristóteles a la discusión de su relación con la felicidad en la ética: véase esp. EN I 8-11.
} 
concretamente a la definición del azar como una causa accidental, que se da, al mismo tiempo, en el ámbito de lo que es "con vistas a algo"

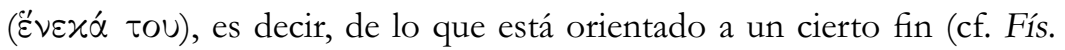
II 5, 196b21-24, 29-31, 197a5-6). En la segunda sección de este trabajo, procuraré explicar en qué consiste para Aristóteles una causa de tipo accidental, lo cual dará la chance de despejar ciertos sobreentendidos sobre ese concepto que surgen, creo, a partir de lo que hoy nos inclinamos a entender a veces como causas accidentales, y permitirá advertir además cuáles son las ventajas sistemáticas de la posición de Aristóteles respecto del problema del azar específicamente.

En la tercera sección, me ocuparé de la segunda característica del azar en sentido genérico (i.e., el hecho de que el mismo se da en el ámbito de lo que es o se produce con vistas a un fin), la cual presenta un par de dificultades sobre las que es preciso decir algo: en primer lugar, hay que reconocer que en un número relativamente importante de pasajes, ${ }^{6}$ el propio Aristóteles presenta tanto a la fortuna como al azar como

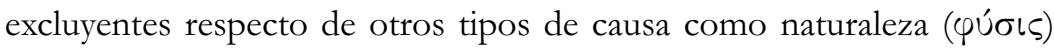
y técnica ( $\tau \dot{\varepsilon} \chi \nu \eta)$, siendo que estos últimos implican, como causas, un componente teleológico; pero, aún más, en AnPo II 11, 95a8-9 la oposición entre azar y teleología parece ser trazada en términos drásticos y poco elusivos: "nada de lo que es por azar sucede con vistas a algo"7 . A pesar de ello, en nuestro texto de Fís. II 5 - y casi por única vez 8 - azar y teleología no sólo no son presentados como mutuamente excluyentes, sino que, por el contrario, el primero parece incluirse en algún sentido en el terreno de la segunda. Y aún más: el darse entre las cosas que son con vistas a algo parece ser, justamente, lo específico o distintivo del azar como causa accidental, i.e. lo que lo distingue de otros tipos de causa

\footnotetext{
${ }^{6}$ Cf. por ej. AnPo II 11, 95a2-9; Met. VII 7, 1032a12-13, 28-30; XII 3, 1070a6-9; Part. Anim. I 1, 640a27-33; Protr. Fr. 11, 5-7 y Fr. 12 (Düring).

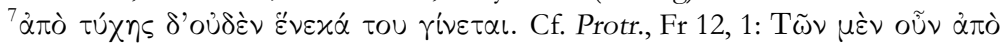

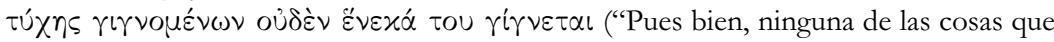
suceden por azar sucede con vistas a algo"). La traducción de los textos es mía.

${ }^{8}$ Cf. además Fís. II 8, 199b18-20.
} 
accidental y le hace merecer un "nombre propio". En segundo lugar, una de las características de aquello que ocurre con vistas a algo es precisamente la regularidad, mientras que una de las notas distintivas de lo accidental consiste en su excepcionalidad ${ }^{10}$. Resulta necesario explicar, pues, en qué sentido lo que es (o podría ser) con vistas a algo puede ser al mismo tiempo accidental, i.e., excepcional y atípico.

Por último, lo anterior me permitirá proponer una interpretación acerca de qué tipo de causa es el azar, es decir, de qué modo corresponde a una de las causas que se presentan en II 3. En primera instancia, dado que Aristóteles afirma repetidas veces en Fís. II 5 que el azar se da en el ámbito de la finalidad, uno tendería a interpretar que el azar es una causa final accidental (así parece entenderlo, por ej., J. Lennox) ${ }^{11}$. Aristóteles, no obstante, en respuesta a este problema, afirma hacia el final de Fís. II 6 que fortuna y azar son causas como aquello de donde proviene el principio del movimiento $(198 \mathrm{a} 2-3)^{12}$. Si bien esto último constituye una afirmación palmaria, no es a mi juicio la respuesta completa acerca del tipo de causa y de fenómeno que es el azar: el punto remanente es cómo se combina ello con la insistencia de II 5 sobre la relación entre azar, accidentalidad y finalidad.

\section{E1 azar es una causa accidental}

El capítulo Fís. II 4, o la introducción al tratamiento del azar, está dedicado a la recopilación y discusión de opiniones sostenidas por anteriores filósofos (y en algunos casos probablemente también por la gente común) respecto del azar como causa. Las opiniones parecen dividirse

${ }^{9} \mathrm{Si}$ se deja de lado esta característica, en efecto, todo lo que nos queda es lo accidental, pero no ya lo azaroso; cf. Ross (1936: 516, ad 196b10-17).

${ }^{10}$ Cf. Met. V 30, 1025a14-21; VI 2, 1026b30-33, 36, 1027a8-12, 15-17; XI 8, 1065a13; Fís. II 8, 199b24-25. La fortuna misma también es por lo tanto esencialmente inestable, Fís. II 5, 197a30-31, cf. EN I 10, 1100b4-7.

${ }^{11}$ Cf. Lennox (1984: 255-56).

${ }^{12}$ Esto viene a responder, en efecto, la pregunta de Fís. II 4, 195b33-34: тív $\alpha$ oũ $v$

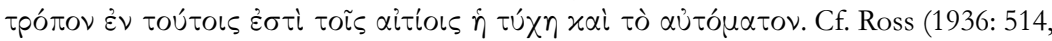
ad 195b33-36). 
esquemáticamente ${ }^{13}$ en dos grandes grupos: por un lado, están quienes niegan que el azar sea causa de cosa o evento alguno (195b36-196a24) y, por el otro quienes, si bien asignan un papel causal al azar, lo restringen sin embargo al plano cosmogónico y lo excluyen del natural sublunar (196a24-b5) ${ }^{14}$. Más allá del interés que posee la evidente y radical inversión que la concepción aristotélica del azar operaría sobre este último esquema (en cuanto pasa a restringir el ámbito de intervención del azar a los asuntos prácticos y la naturaleza a nivel sublunar, excluyéndolo en cambio del ámbito cosmológico), ${ }^{15}$ me interesa reparar aquí en el primer argumento considerado y discutido por Aristóteles, en 195b36-196a7, el cual cuenta en contra de la existencia del azar como causa. La razón en la que este argumento se apoya para concluir que no hay tal cosa como el azar, es que siempre es posible encontrar alguna causa de lo que decimos que se produce por azar. Así, la causa de haberse encontrado en el mercado con quien quería ver es querer ir al mercado a comprar algo; la causa de que uno fuera al lugar en donde le esperaba una muerte violenta es querer salir a tomar agua luego de una cena demasiado condimentada;

\footnotetext{
${ }^{13}$ Además de las que mencionaré, Aristóteles agrega hacia el final del capítulo en unas pocas líneas la opinión que sostiene que el azar es algo oculto al entendimiento humano por ser algo divino (196b5-7). Esta opinión correspondería probablemente a la concepción más popular del azar, cf. infra nota 29.

${ }^{14}$ Si bien Aristóteles presenta ambas tesis por separado, y afirma que, según la pri-

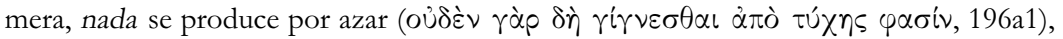
Simplicio (cf. In Phys., 330.13-20, cf. Ross, 1936: 514, ad 195b36-196a3) atribuye tanto esta tesis como la segunda — combinándolas - a Demócrito, en la medida en que la primera se referiría solamente al ámbito práctico. Hay que reconocer que, además de este testimonio de Simplicio (= DK 68A68), existen ciertos fragmentos que permitirían atribuir una postura semejante, respecto del ámbito práctico, al filósofo de Abdera; cf. por ej. DK 68B118.

${ }^{15}$ Una inversión que tendrá a su vez consecuencias de peso en el modo de pensar la relación entre azar y teleología en relación a la tradición precedente. En efecto, una de las razones que, a mi juicio, permiten a Aristóteles no presentar en Fís. II 5-6 al azar y a la teleología como mutuamente excluyentes —una relación que habría quedado así planteada fundamentalmente desde el Timeo de Platón- es el hecho de no tratarlos como principios o fuerzas de orden cosmológico (196b1-4) ni mucho menos cosmogónico - esto último no sólo por las razones de principio expuestas en 198a10-13, sino además porque, como se sabe, Aristóteles defiende la tesis de que el universo es ingenerado (DC I 10-12).
} 
e incluso, la causa de que Colón llegara a América es que partió rumbo a las Indias. Por lo tanto, de ninguno de estos eventos es causa el azar. Más allá de que se podría objetar, en todos estos casos, si realmente lo que se aduce como causa es propiamente causa del resultado mencionado, es interesante notar el supuesto sobre el que se apoya este argumento, a saber: que la existencia de eventos azarosos implicaría la negación de lo que, en términos modernos, llamaríamos el principio de razón suficiente. Dicho de otro modo: el argumento supone que si en verdad hay eventos que suceden por azar, entonces hay eventos que carecen de causa, dado que si es posible señalar una causa para los mismos, ello excluye la posibilidad de que hayan ocurrido por azar. El azar implicaría entonces, si existe, una suerte de agujero causal ${ }^{16}$.

Aristóteles responde al argumento rechazando por elevación el supuesto sobre el que se apoyan los atomistas como un non sequitur: la posibilidad de señalar algo puntual como causa de un evento determinado no excluye la posibilidad de que ello haya sucedido, no obstante, por azar. Todos opinan, de hecho, que cierto tipo de cosas suceden por azar a pesar de que tienen una causa (196a15-16), y este ع́ $v \delta \circ \xi o \nu$ tiene, a juicio de Aristóteles, algún sustento. Lo que permite a Aristóteles desbrozar una vía de salida de la dicotomía causa-azar, y con ello afirmar al mismo tiempo que hay eventos que suceden por azar y que es posible indicar la causa de cada uno de ellos, es por un lado la distinción entre varios sentidos de causa (asunto que retomaré más adelante) y por el otro el concepto de causa accidental. En efecto, los eventos que ocurren por azar tienen siempre una causa, pero lo peculiar es que se tratará siempre, en estos casos, de una causa accidental. Ahora bien, para comprender

\footnotetext{
${ }^{16}$ Es interesante notar, al margen de la opinión atomista, que el argumento por recurso al principio de razón suficiente (o la ausencia de eventos incausados), es uno de los argumentos esgrimidos por varios autores que han defendido, desde puntos de partida y con matices bien diferentes, tanto la idea de que el azar es una causa meramente imaginaria y carente de consistencia ontológica ("un nombre vacío" como parafrasea Temistio, cf. In Phys. 47.17), como la tesis del determinismo causal. Entre estos filósofos, podemos mencionar, por caso, desde los estoicos — ver esp. Alejandro de Afrodisias en el De Fato 192.8-15 (= Long-Sedley 55N, 11-18 y cf. Ib. vol I., p. 343)— hasta Laplace (1820: vi).
} 
qué significa esto para el caso del azar debemos revisar en qué consisten en general, según Aristóteles, las causas accidentales.

Este tipo de causa es presentado en 195a32-b3, dentro de la sección de Fís. II 3 en donde Aristóteles distingue los modos en que las causas pueden darse o enunciarse en una proposición (195a26 ss.); pues bien, uno de los modos de enunciar la causa de algo consiste precisamente en hacerlo mediante una descripción bajo la cual ella resulta meramente accidental respecto de lo causado, o mejor: en cuanto (qua) causa de aquello de lo cual es causa. Tenemos cierta tendencia hoy día a entender bajo "causa accidental" un evento o suceso que ocurre él mismo de modo accidental o incausado y que (se supone) tendrá a su vez una serie de efectos en el mundo. No obstante, para Aristóteles, la causa accidental no constituye una suerte de entidad o potencia salida de la nada que inaugura una cadena causal en una dirección cualquiera (imprevista o imprevisible a partir del estado actual del mundo), ${ }^{17}$ sino que representa más bien un cierto modo de describir una relación causal dada en una proposición causal, es decir, en una proposición que expresa una relación existente entre un término que se refiere a la causa y otro término que se refiere a aquello de lo cual la causa es causa. La causa accidental, como se verá un poco más claro en seguida, es en todo caso accidental qua causa de determinado efecto, y no accidental a secas. En este punto es preciso detenerse para subrayar una serie de cuestiones.

\footnotetext{
${ }^{17} \mathrm{~A}$ mi entender, este tipo de esquema trasluce por ejemplo en ciertas interpretaciones de Met. VI 3, otro de los textos — por demás oscuro- en que Aristóteles se ocupa de las causas accidentales y en el que afirma entre otras cosas que la búsqueda de las causas de un determinado evento no puede remontarse al infinito y debe detenerse en algún punto, el cual consistiría, presumiblemente, en una causa de tipo accidental que carece, ella misma, de causa. Me refiero, concretamente, a las interpretaciones que entienden esta causa accidental como "inaugural" en el sentido arriba mencionado, e intentan rastrearla en una decisión producto de la voluntad de un agente, la cual no sería a su vez causada necesariamente por ningún evento antecedente en el mundo sino que sería "espontánea” y permitiría a su vez iniciar nuevas cadenas causales en el mundo (cf. Ross, 1924: lxxx; Madigan, 1984: 129). Esta lectura pasa por alto el punto fundamental que arriba señalo: las causas accidentales son cierto tipo de descripciones de relaciones causales que ya se dan de hecho en el mundo y no eventos o cosas salidas de la nada.
} 
1) Como se deja ver a partir de lo anterior, la distinción entre causa "accidental" y "por sí" se da a nivel explicativo o proposicional. La causa accidental es un cierto modo de describir una relación causal que, bajo otra descripción, es en cambio "por sî". Así, al decir "el constructor es la causa de la casa" se da la causa por sí de esta última, mientras que "el blanco es la causa de la casa" expresa en cambio la causa accidental; supuesto que el constructor sea además blanco (cf. 196b26-29). Lo mismo vale para aquello que de lo cual la causa es causa (195b6-7) — el efecto ${ }^{18}$ — lo cual admite también, por supuesto, diferentes descripciones. Aún más, que la relación causal expresada en la proposición sea accidental o por sí depende no sólo de la descripción de la causa sino también y en igual medida de la descripción de lo causado; en efecto, no hay causa a secas, sino siempre causa de algo. En definitiva, en una proposición causal "A es causa de B", el "es causa de" ha de entenderse en sentido accidental o bien por sí dependiendo de qué ocupe el lugar de A y de $\mathrm{B}$ en dicha proposición; lo cual debe combinarse a su vez con uno de los cuatro sentidos principales en los que "causa" puede entenderse.

Esto implica que una misma entidad puede ser causa por sí o accidental dependiendo de cómo se describa lo causado: ${ }^{19}$ en "el cocinero es

\footnotetext{
${ }^{18}$ Prefiero evitar en la medida de lo posible — como el lector advertirá- esta denominación. Por un lado porque, como es sabido, Aristóteles no emplea en griego una palabra equivalente, sino que habla en todo caso de aquello de lo cual la causa es causa, i. e. aquello que la causa viene a explicar; y, por otro lado, porque lo anterior no es una cuestión meramente terminológica: creo que actualmente "efecto" tiene la connotación de un resultado futuro respecto de la causa, y a veces incluso desconocido en el presente en que la causa ocurre, mientras que para Aristóteles el caso típico de relación causal es aquel en el que causa y "efecto" son simultáneos (AnPo II 12, 95a10-14, 22-24) y, aún más, lo causado lejos de ser un resultado incierto es aquello de lo cual se parte en la investigación de las (sus) causas (cf. AnPo II 1, 89b29-31). Véase para este punto Wieland (1972: 52-53).

${ }^{19}$ Simplicio entiende también que en estos casos una y la misma acción puede ser causa por sí de una cosa y causa accidental de otra: cavar un pozo es la causa per se de forestar, pero es la causa per accidens de encontrar el tesoro (Simplicio In Phys. 337.19-27); Alejandro de Afrodisias también parece haber sostenido algo semejante (cf. Simplicio In Phys. 343.17-18). Sorabji (1980: 3-25) trata estos casos (que llama "coincidences"), en los que la descripción relevante del 'efecto' carece de causa por sí, como casos de un tipo diferente de aquellos en que la causa se enuncia bajo una descripción
} 
la causa de esta sabrosa cena" el cocinero es la causa por sí, mientras que, si la cena es además saludable, "el cocinero es la causa de este alimento saludable" expresa en cambio una relación causal de tipo accidental (pues el arte culinaria tiene por objeto lo agradable, y no lo saludable). En este caso, en efecto, hay cierta descripción de lo causado que carece de una causa por sí, o como dice Aristóteles: que no es alcanzado en cuanto ello mismo, sino en cuanto otra cosa (Met. V 30, 1025a28-29): el alimento saludable es elaborado en cuanto sabroso, se llega a América en cuanto lugar hacia el que no se navegaba (cf. 1025a29-30); y uno va allí donde está quien le debe dinero, pero en cuanto va a ver un espectáculo, o a ver a alguien, etc. (cf. Fís. II 5, 197a15-18). En suma: un proceso culmina en un punto hacia el cual no estaba dirigido; o incluso, para aquellos casos que involucran la dimensión práctica: una acción se realiza bajo una descripción que resulta, a la postre, no ser la más relevante. A este tipo de esquema corresponde justamente el azar como relación causal accidental. Pero dejo esto por un momento.

2) Todo esto no implica que para Aristóteles las causas sean entidades lingüísticas, ni los contextos causales necesariamente intensionales, por más que las causas sean interpretadas como explicaciones. Las causas no dejan de ser cosas reales en el mundo, entidades a las que se apela para dar cuenta de cosas o eventos: ${ }^{20}$ propiedades, capacidades o potencias, procesos, sustancias, etc.; pero, al mismo tiempo, es cierto que estas causas se expresan en proposiciones, al interior de las cuales pueden aparecer bajo diferentes descripciones. Ahora bien - y este es un punto fundamental — si las causas y las cosas causadas por ellas admiten diferentes descripciones, es porque ellas son parte de entidades com-

accidental. La diferencia más saliente, según el autor, es que las "coincidencias" carecen de causa. En todo caso, no considero que haya razones de peso para tratar este tipo de relación causal accidental como diferente de la primera: el propio Aristóteles trata una y otra de modo similar y no traza, hasta donde veo, distinciones de semejante peso entre ellas. La tesis más fuerte de Sorabji, según la cual las “coincidencias" carecende causa por carecer de explicación, ha sido harto discutida por los intérpretes, y el espíritu general de esta sección se aparta de ella, aunque en el apartado final se verá que en cierto sentido (más concretamente, para uno de los sentidos de "causa") Sorabji está en lo cierto.

${ }^{20}$ Freeland (1991: 52) sostiene en este sentido que Aristóteles es un realista explicativo; Moravcsik (1991: 31-32) habla de una teoría correspondentista de las explicaciones. 
plejas, esto es: compuestos en los que coinciden infinitos predicados en una sustancia (196b28-29) ${ }^{21}$. Dicho de otro modo, la base ontológica que posibilita y hace verdaderas las múltiples descripciones de causa y efecto en las proposiciones causales es la existencia de unidades accidentales. De aquí se sigue, a mi juicio, que la posibilidad de realizar infinitas descripciones de una relación causal no resulta incompatible con la extensionalidad de los contextos causales en Aristóteles. En las proposiciones causales aristotélicas, en efecto, las diferentes descripciones de causa y efecto son, en cierto sentido, correferenciales, pues se refieren siempre a una misma unidad (accidental), que es la que aglutina las diversas entidades que se mencionan cada vez en las diferentes descripciones ${ }^{22}$. Y además, fundamentalmente, estas descripciones correferenciales son sustituibles en dichas proposiciones sin alterar el valor de verdad de las mismas, sino sólo el alcance con que "causa" debe entenderse. "El escultor es la causa de la estatua" es tan verdadero como "Policleto es la causa de la estatua", dado que Policleto es justamente el escultor; sólo que lo primero es verdadero no sólo en este caso particular sino además siempre o la mayoría de las veces, y lo segundo sólo en este caso particular $^{23}$. Pero volveré a esto último un poco más abajo. Por ahora, conviene subrayar una vez más que la causa accidental o por accidente

\footnotetext{
${ }^{21}$ De allí que las causas accidentales pueden ser infinitas en número (197a15-17).

${ }^{22} \mathrm{Si}$ bien estoy de acuerdo con Freeland (1991: 54) en que, por caso, "Policleto", "el hombre" y "el escultor" no son estrictamente correferenciales, pues constituyen, para Aristóteles diferentes entidades, considero sin embargo que no puede dejar de reconocerse que son correferenciales en cierto modo, justamente por estar todas estas entidades aglutinadas en una unidad que supone, además, una entidad sustancial como sustrato (cf. Met. V 6, 1015b16-34 y Ross, 1924 I: 301, ad loc.). Si la ausencia de correferencialidad fuera absoluta, no podría decirse siquiera con verdad que Policleto es causa accidental de la estatua, sino que daría lo mismo decir que un caballo lo es.

${ }^{23} \mathrm{El}$ concepto de causa en Aristóteles no parece requerir, en efecto, la existencia de una ley universal o englobante de la cual la proposición causal particular sea una instancia. Este último requisito es, por el contrario, más bien propio de lo que usualmente se considera la concepción humeana de la causalidad (en Aristóteles por el contrario no hay indicaciones claras de que el concepto de causalidad implique el de ley, ni que las explicaciones sean nomológico deductivas). De aquí se sigue que para Aristóteles el concepto mismo de explicación (si se considera equivalente al de causa) no tiene por qué restringirse al ámbito estrictamente científico en donde sólo son admisibles propo-
} 
no es menos "real" que la causa por sí, y que la proposición en que se expresa es una proposición causal verdadera: baste recordar que el accidente es "aquello que se da en algo y su enunciación es verdadera, pero, desde luego, ni necesariamente ni la mayoría de las veces" 24 .

3) Es importante acentuar que de lo expuesto se sigue que la causa accidental es accidental en cuanto causa de determinado efecto. La cualificación es fundamental, y es necesario insistir lo suficiente en ella para evitar el riesgo de confusiones conceptuales muy a la mano y que desvían irremediablemente la comprensión del planteo aristotélico. Me refiero al riesgo de tomar la causa accidental como equivalente al accidente en sentido ontológico, es decir, identificar la distinción entre las causas por sí (o causas en sentido propio) y las causas accidentales con la distinción categorial entre sustancia y accidentes. Lejos de ello, una entidad que pertenece a una categoría accidental puede bien funcionar, según el caso, como causa accidental o bien como causa por sí; y una sustancia puede, así mismo, ser una causa accidental. Lo relevante en estos casos es más bien, como he intentado mostrar, la relación que guarda la entidad mencionada como causa con la entidad mencionada como lo causado en la proposición. Es en función de esa relación que el calificativo "accidental" ( $x \alpha \tau \grave{\alpha} \sigma \cup \mu \beta \varepsilon \beta \eta x o ́ \varsigma)$ se aplica en este tipo de contexto, y es por eso que en este caso vale decir que a aquello que constituye la causa propia o por sí, le acaece accidentalmente ser además infinitas cosas que, respecto de su carácter de causa de una determinada cosa o evento, resultan accidentales (cf. 196b28-29, 195a34-35).

4) Quisiera reparar, brevemente, en ciertos aspectos de la diferencia entre la causa por sí y la causa accidental. Por lo que hemos visto, conviene no apoyarse linealmente en la diferencia categorial sustanciaaccidente para atacar este problema; en cambio, una forma posible de encararlo es preguntarse en cada caso de qué modo lo mencionado como causa (A) en la proposición resulta explicativo respecto de lo que se menciona como causado por ella (B), i.e. qué alcance tiene la rela-

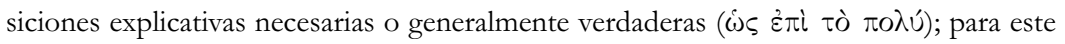
último punto véase también Everson (1988: 53-56).

${ }^{24}$ Met. V 30, 1025a14-15; el subrayado es mío. 
ción causal explicativa entre A y B. En esta línea, Kirwan (1993: 182) sostiene que la causa por sí es "self-explanatory" respecto de lo causado; Sorabji (1980: 11) por su parte traduce esta distinción entre ambos tipos de causa-explicación en términos de lo que es directa o indirectamente explicativo respecto de lo causado ${ }^{25}$. Ambos autores apuntan al hecho de que, cuando se enuncia la causa por sí de algo, no es necesario apelar a ninguna otra cosa para aprehenderla como causa de lo causado: ella lo explica por sí misma ${ }^{26}$. El escultor es causa de la estatua, porque el arte de esculpir es, desde un punto de vista conceptual y por naturaleza (cf. 1027a1-2), la potencia o capacidad de producir el tipo de cosa que son las estatuas; y, por lo tanto, si alguien produce una estatua lo hace necesariamente en cuanto (qua) escultor, sean cuales fueren sus demás atributos. La causa accidental, en cambio, no es explicativa en este sentido autosuficiente o directo respecto de lo causado, sino en un sentido indirecto y aparentemente restringido. ¿En qué consiste esta restricción? La respuesta que se suele dar es la siguiente: en "Policleto es causa de la estatua", Policleto sólo explica la producción de la estatua en la medida en que él es escultor (y esto último, a su vez, ya explica por sí mismo la estatua), ${ }^{27}$ de modo que la causa accidental explica lo causado en la medida en que se apoya, por así decir, en la causa por sí; pero - $\mathrm{y}$ este es un punto importante - no todo escultor es Policleto, sino que meramente se da el caso particular de que éste lo es, i.e. ocurre accidentalmente que el escultor es Policleto (195a34-35). Podría decirse, a partir de esto, que la causa por sí es explicativa respecto de lo causado independientemente del contexto: la proposición causal que expresa una

\footnotetext{
${ }^{25}$ Como he mencionado antes, este autor no considera sin embargo que en el caso del azar y en general de lo que llama "coincidencias" haya siquiera explicación indirecta (i.e. causa accidental), cf. supra nota 19.

${ }^{26}$ Para otro modo de entender la diferencia entre estos dos tipos de causa, véase D. Frede (1992) quien interpreta la diferencia entre lo que es accidental y por sí en términos de lo que cae dentro o fuera de la determinación teleológica; cf. en la misma línea Natali (1999).

${ }^{27}$ Cf. Sorabji (1980: 11). Del mismo modo podría decirse (aunque Sorabji no estaría de acuerdo con esto) que el haber querido ir al mercado para ver a alguien explica por qué tal persona ha encontrado a su deudor, en la medida en que explica (por sí mismo) por qué fue al mercado, en donde accidentalmente se encontraba su deudor.
} 
causa por sí es verdadera en todos o casi todos los contextos de uso (i.e. siempre o la mayoría de las veces) ${ }^{28}$. En cambio, la causa accidental (y el azar por lo tanto) remite siempre a un contexto determinado, es decir: se trata de una proposición causal que involucra una descripción tal de la causa y de lo causado que resulta explicativa sólo en cierto contexto de uso. En otras palabras, se trata de una proposición que es verdadera en el caso particular, pero no siempre ni la mayoría de las veces. Policleto es la causa de la estatua en este caso, pero no en todos, y esto porque en este caso el escultor resulta ser Policleto. Nuevamente, la diferencia entre causa accidental y causa por sí no es una que apunte a distinguir entre una causa más verdadera o más real que otra, sino más bien entre dos descripciones de una relación causal que aportan explicaciones de diferente alcance: una con alcance científico, verdadera para todos o casi todos los contextos, en cuanto rescata lo que hay de esencial en la relación causal, y la otra verdadera sólo para el caso particular.

5) Para terminar esta sección, conviene revisar qué implica lo dicho hasta aquí para el problema del azar, dado que aquel es un cierto tipo de causa accidental. En primer lugar, entender el azar como una causa accidental implica "descosificarlo", si se interpretan las causas accidentales como cierto tipo de relaciones causales. Así pues, fortuna y azar no son causas en el sentido de fuerzas o potencias causales cósmicas (como parecen sugerir anteriores filósofos) ni que produzcan efectos "desde bambalinas" en el curso de los acontecimientos humanos (como podría sugerir la concepción vulgar del azar que llega incluso a personificarlo), ${ }^{29}$ y por ello, además, es que la existencia del azar no resulta incompatible con la posibilidad de señalar una causa determinada de lo ocurrido en

\footnotetext{
${ }^{28}$ Esto es coherente con la tesis aristotélica de que la ciencia demostrativa se ocupa de las causas por sí y no de las causas accidentales, puesto que ella tiene que ver con

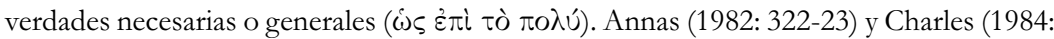
48-49) interpretan en efecto la causa por sí de Fís. II como aquella descripción que puede funcionar como término medio de un silogismo demostrativo.

${ }^{29}$ Cf. 196b5-7, pasaje que según Simplicio refiere justamente a la opinión común del vulgo en Grecia, incluso en tiempos anteriores a Aristóteles, y que se manifiesta en el culto a Tú \ク como una divinidad (Simplicio, In Phys., 333.5-17; cf. Ib. 360.27-361.1 sobre las tradiciones y representaciones populares de la diosa).
} 
cada caso particular, por más que se trate de una causa accidental. En segundo lugar, es importante notar que a diferencia de lo que hoy día se tiende a concebir por "azar", éste no es para Aristóteles, de acuerdo al planteo de Fís. II 4-6, aquel estado de cosas cuyo resultado futuro no puede conocerse con certeza - por los motivos que fuere-, sino que azaroso es más bien aquel resultado ya alcanzado de hecho, pero que es inexplicable desde el punto de vista científico o tipológico; dicho de otro modo: es aquel evento que carece de causa por sí y sólo tiene causas accidentales, lo cual implica tanto como decir que ellas valen (como causas de ese resultado) sólo en el caso particular, pero que, consideradas desde un punto de vista tipológico, son potencialmente infinitas (y de allí que

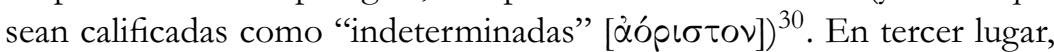
puesto que en el fenómeno del azar lo peculiar parece ser que no hay causa por sí del efecto bajo su descripción más relevante, cabe preguntarse, de entre las infinitas descripciones posibles que admite el efecto, qué es lo que hace a una descripción relevante y no a otra, como para preguntar por su causa. Pues bien, la respuesta a este último interrogante se halla, a mi entender, en la segunda nota definitoria del azar, i.e., el darse en el ámbito de lo que es con vistas a algo.

\section{Azar y teleología}

La relación entre azar y teleología, problemática como es, ha sido interpretada de diferentes maneras por los estudiosos de Aristóteles; desde quienes entienden que se trata de una oposición tajante (una interpretación que en general va asociada con la idea de que la teleología es un principio de alcance cosmológico, y que ha sido defendida en los comienzos del siglo pasado sobre todo), ${ }^{31}$ hasta quienes consideran que

\footnotetext{
${ }^{30}$ Cf. Fís. II 5, 196b27-28, 197a20-21; Met. V 30, 1025a24-25; VI 4, 1027b33-34; XI 8, 1065a25; APr I 13, 32b4-11; Protr. Fr. 12, 11. Hasta donde entiendo, la calificación de "indeterminadas" se predica de las causas accidentales sólo como tipo, y no distributivamente.

${ }^{31}$ Loening (1903: 155-56 n. 57, 160-61); A. Mansion (1913: 179-188); Zeller (1921: 330 ss., 427 ss.). Cf. supra nota 15. Para una lúcida e instructiva crítica a este tipo de lecturas, véase Wieland (1962: 255-59).
} 
azar y teleología se encuentran estrechamente ligados, interpretación que goza hoy día de mayor aceptación, pero que ofrece a su vez variantes y matices. Lo que propondré aquí es una interpretación en esta segunda línea, es decir, que intenta buscar los puntos de contacto entre azar y teleología ${ }^{32}$.

Retomando el último punto del apartado anterior, podemos preguntarnos qué es lo que hace que la acción relatada en el ejemplo de Fís. II 5 (196b33 ss.) sea descripta como "toparse con quien le debe dinero", lo cual carece de una causa por sí, y no como, simplemente, "ir a, o estar en, el mercado". O incluso, por qué la misma acción no se describe como "llegar a las 15:30 al mismo lugar por el que pasó Sócrates ayer", lo cual, de hecho, carece también de causa por sí, y sin embargo no calificaríamos como azaroso a primera vista. Piénsese incluso en la posibilidad hipotética de que nuestro personaje simplemente pasara de largo al lado de su deudor (sin dejar de verlo) al no reconocer la situación como una oportunidad de recobrar su dinero. ¿Contaría éste de todos modos como un caso de azar? A mi juicio, la respuesta es "no". No apunto con esto a que la descripción relevante dependa de lo que uno u otro sujeto particular reconoce como relevante: más bien, para advertir qué es lo que hace relevante a una descripción particular del proceso en cuestión y no a otra, hay que prestar atención a la segunda característica general del azar según la definición de Aristóteles, esto es: el darse en el ámbito de lo que es con vistas a algo. Si el resultado del proceso es relevante bajo cierta descripción, es porque esa descripción corresponde a algo que

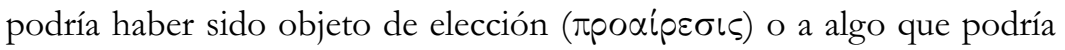
haber ocurrido por naturaleza (cf. Fís. II 5, 196b21-22). A estas dos cau-

\footnotetext{
${ }^{32}$ Dentro de esta segunda línea de interpretación, la lectura que expondré a continuación va, fundamentalmente, en la misma senda que las de Wieland (1962), Lennox (1984), en cierto modo Charlton (1992) y Simplicio. Lo que tienen en común estas lecturas es que consideran que el punto de contacto entre azar y teleología debe buscarse en el resultado del proceso azaroso. De manera un tanto diferente lo entiende Boeri (1995), quien está más bien de acuerdo con Porfirio (según Simplicio, In Phys., 336.27-29, cf. Lennox, 1984: 251-254) en poner el acento de la relación entre azar y teleología en el hecho de que la acción que desemboca en un resultado azaroso era ella misma con vistas a algún fin (diferente del alcanzado de hecho).
} 
sas habría que agregar aun lo que podría haber sido producido mediante la técnica. Todas estas son causas, precisamente, de procesos teleológicamente orientados, procesos en los cuales el principio del movimiento que les da origen conlleva una determinación o componente formal-final que, como causa, lo orienta en determinado sentido e impone un cierto orden de antero-posterioridad en la sucesión de sus etapas (cf. Fís. II 8, 199a8-20) ${ }^{33}$. Pero volveré a esto en seguida.

El resultado del proceso azaroso es entonces, bajo su descripción relevante, el tipo de cosa que constituye normalmente la causa final de un proceso, y sin embargo, en el caso puntual del azar, es alcanzado sin constituir la causa (final) del proceso que le dio lugar: lejos de ello, el resultado parece apoyarse en un proceso que no estaba dirigido a él, ni fue causado por él como fin. La distinción que aquí se perfila y que resulta decisiva para entender este texto de la Física — como ha mostrado Ross (1936: 518) y más recientemente Lennox (1984) — es la que existe entre el fin (o 'aquello con vistas a lo cual') como resultado de un proceso y causa del mismo, y el fin como mero resultado de un proceso pero sin valor causal respecto del mismo. El proceso azaroso, por lo dicho, no puede ser explicado (ni siquiera de modo accidental) a partir de este resultado ${ }^{34}$. Ahora bien, si esto es así, ¿̇por qué razón afirma de todos modos Aristóteles que el azar se da en el ámbito de lo que es con vistas a algo? Hay que dar, en principio, dos respuestas a esta pregunta: una de orden terminológico, y una de orden sistemático.

En el plano terminológico, lo cierto es que Aristóteles usa las mismas expresiones para referirse a lo genuinamente teleológico y a lo aparentemente teleológico, y al resultado alcanzado de hecho y a la causa final

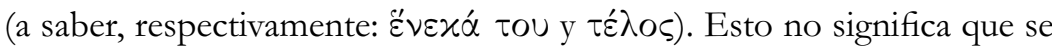
trata aquí de una ambigüedad meramente accidental, pero permite interpretar al menos coherentemente un conjunto de pasajes de Fís. II 5 que de otro modo resultan abiertamente contradictorios ${ }^{35}$. En definitiva, lo

\footnotetext{
${ }^{33}$ Sobre la relación estrecha entre la causa final y el orden en la sucesión de las etapas del proceso causado por ella, véase Charles (1991).

${ }^{34}$ Cf. Lennox (1982: 238).

${ }^{35}$ Cf. por ejemplo, 196b34-36, en donde Aristóteles pasa de un sentido al otro de

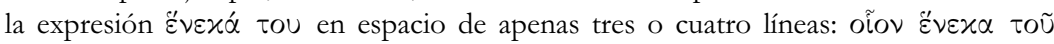


que esta distinción de sentidos implica es que cuando Aristóteles dice que el azar se da en el ámbito de lo que es con vistas a algo, no está usando esta última expresión en su sentido más restringido y estricto, sino en el sentido más amplio de aquello que es y también aquello que podría ser con vistas a un fin, es decir, en un sentido que incluye tanto los resultados que poseen y los que no poseen valor causal.

Ahora bien, más allá de estas cuestiones terminológicas, si el azar constituye un caso de teleología aparente y no genuina, no es menos cierto que la apariencia de finalidad sólo puede darse en un ámbito en el cual se dé también al mismo tiempo (y típicamente) la genuina finalidad; en otras palabras, la apariencia de finalidad sólo puede darse allí donde sería esperable una cierta finalidad, o en términos aristotélicos: en el ámbito de lo que es "con vistas a algo". Viendo esto mismo desde el punto de vista de la causalidad podría decirse lo anterior del siguiente modo: si bien es cierto que azar y finalidad se excluyen mutuamente como causas, ${ }^{36}$ esto sólo puede sostenerse para las cosas o eventos en cuanto individuales, i.e. dado un evento o una cosa particular, ello sólo pudo haber sido causado (a) con vistas al resultado que se alcanzó de hecho o bien (b) por azar, pero no por ambas causas: este individuo se encontró con su deudor (a) a partir de su elección o (b) por azar, pero no por ambas causas. Si nos situamos en cambio en el plano tipológico y no particular, hay que sostener exactamente lo contrario: hay ciertos tipos de eventos y de cosas que pueden ser producidos tanto con vistas a un fin como por azar (el tipo de evento consistente en encontrarse con

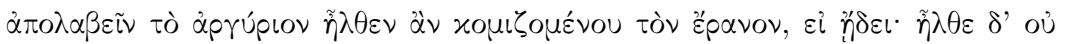

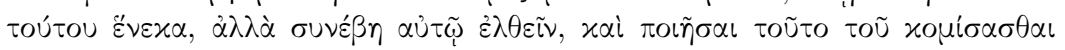

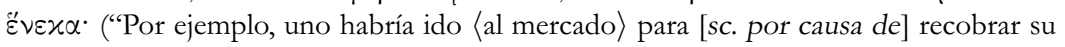
dinero, en el momento en que su deudor obtendría un pago, si hubiera sabido <esto último $\rangle$; pero no fue para esto, sino que sucedió que fue, y que lo hizo para [sc. con el resultado de] recobrar (el dinero〉.”). En 197a1-2, y siguiendo con el mismo ejemplo del

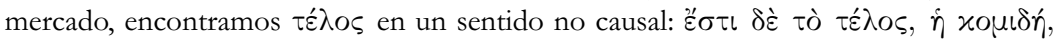

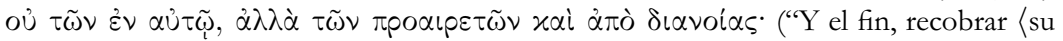
dinero〉, no está entre las causas 〈presentes〉 en él 〈para ir al mercado〉, pero es una de las cosas elegibles y que son a partir de un propósito”).

${ }^{36}$ Cf. supra en la nota 6 los pasajes en donde Aristóteles en efecto establece esta oposición en términos excluyentes. 
quien le debe plata puede darse tanto por elección como por azar), i.e. desde este punto de vista, azar y finalidad no sólo no se excluyen, sino que comparten el mismo ámbito. La compatibilidad de azar y finalidad, entonces, debe entenderse a mi juicio desde un punto de vista tipológico, el cual a su vez no está desconectado de su exclusión mutua desde el punto de vista particular: muy lejos de ello, lo primero constituye más bien la condición de esta oposición excluyente.

\section{A modo de conclusión}

Para concluir, si lo anterior es correcto y el azar constituye una suerte de teleología aparente pero no real, hay que dar entonces la razón a Sorabji ${ }^{37}$ en cierto sentido: lo que ocurre por azar, o como dice Sorabji, las "coincidencias", carecen de causa si "causa" se entiende como 'aquello con vistas a lo cual', o el fin. Pero, dado que "causa" se entiende en más de un sentido, Aristóteles puede sostener al mismo tiempo que lo que ocurre por azar carece de causa (i.e. final) y que tiene una causa (i.e. como aquello de donde proviene el principio del movimiento) que es a su vez accidental, con lo cual nuestro filósofo está en condiciones de reconocer la existencia del azar sin negar por ello que todo lo que ocurre tiene una causa, dando por tierra anticipadamente con algunos argumentos deterministas en contra de la existencia del azar ${ }^{38}$.

Por su parte, el carácter accidental de la llamada causa eficiente en el caso del azar parece consistir, precisamente, en que ella no está orientada originalmente por un fin correspondiente al resultado que de hecho se obtiene al cabo de proceso: es por eso que el resultado (bajo su descripción más relevante) se encuentra en una relación causal accidental con el principio del movimiento que lo produjo en el caso particular. En los casos normales, en cambio, la causa como aquello de donde proviene el principio del movimiento y la causa como forma y como fin, coinciden o son una (Fís. II 7, 198a24-26); esto es: la causa eficiente comporta una determinación formal-final (cf. Ib. III 2, 202a9-12) que es la causa de que

\footnotetext{
${ }^{37}$ Cf. supra nota 19.

${ }^{38}$ Cf. supra nota 16 .
} 
el proceso iniciado por ella siga un determinado orden y esté direccionado de cierto modo y no de otro ${ }^{39}$. Esto vale tanto para la generación de los seres naturales ${ }^{40}$ como en el caso de lo que se produce por causa de la

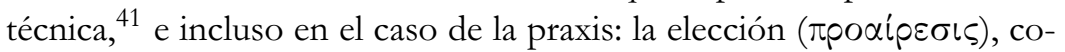
mo aquello de donde proviene el principio del movimiento que da lugar a la acción implica necesariamente un componente final, pues, como determinación de los medios, tiene lugar y se encuentra orientada siempre a partir de un fin deseado (cf. EN VI 2, 1139a31-33). Puede pensarse, en tal medida, que el azar — sea en el ámbito natural, en el de la técnica o en el de la praxis - es un fenómeno en el cual la causa en el sentido de 'aquello de donde proviene el principio del movimiento' es accidental porque carece de la determinación causal formal-final correspondiente al resultado del proceso: en el azar hay un desfase entre aquello que determina formal-finalmente al motor de un proceso y el resultado de ese proceso $^{42}$.

En definitiva, la respuesta a la pregunta por el modo en que el azar encaja en el esquema de las causas de Fís. II 3 es, sin duda, que el mismo es una causa accidental en el sentido de aquello de donde proviene el principio del movimiento (Fís. II 6, 198a2-3). Ahora bien, respecto de la pregunta acerca de qué es el azar (y no ya sólo qué tipo de causa es), lo anterior constituye sólo la mitad de la respuesta. En efecto, el azar posee también un aspecto no causal, y en esto consiste la segunda parte de su definición: el emular aquello que es con vistas a un fin, sin ser no obstante una genuina causa final. Este aspecto del azar, su carácter de finalidad aparente, no refleja ya su naturaleza en cuanto causa, y en tal sentido permanece irreductible al esquema de Fís. II 3.

\footnotetext{
${ }^{39}$ Esto es lo que explica que los seres vivos se generen en cada caso a partir de un homónimo (Met. VII 7, 1034a22-23, cf. Fís. II 7, 198a26-27).

${ }^{40}$ Cf. Gen. Anim. I 20, 729a9-10.

${ }^{41}$ Cf. Met. VII 7, 1032a32 ss., VII 9, 1034a21-26; Gen. Anim. II 4, 740b25-29.

${ }^{42}$ Esto puede verse ilustrado claramente en lo que estipula Aristóteles respecto de las técnicas o artes y el azar: puede haber azar sólo en las técnicas que operan sobre una materia capaz de moverse de modo determinado a partir de un principio interno, cf. Met. VII 9, 1034a9-21; cf. AnPo II 11, 95a3-6. Esto es: allí donde la materia es capaz de moverse a partir de otro motor diferente de la técnica, pero produciendo el mismo resultado que ella, allí es posible el azar.
} 


\section{Referencias}

AnNAS, J. (1982): "Aristotle on inefficient causes", Philosophical Quarterly 32 (1982), pp. 311-326.

Boeri, M. (1993): Aristóteles, Física. I y II, traducción, introducción y comentario por Marcelo D. Boeri, Buenos Aires: Biblos, 1993.

- (1995): “Chance and Teleology in Aristotle's Physics", International Philosophical Quarterly, Vol. XXXV, No 1, pp. 87-96.

Charles, D. (1984): Aristotle's Philosophy of Action, New York: Ithaca, 1984.

- (1991): "Teleological causation in the Physics", en L. Judson (ed.) (1991), pp. 101-128.

Charlton, W. (1992): Aristotle, Physics Books I and II, Translation, Introduction, Commentary by W. Charlton, Oxford, 1995 (=1992, $\left.1970^{1}\right)$.

Diels, H. (ed.): Simplicii in Aristotelis Physicorum libros quattuor priores commentaria, CAG,Vol. IX, Berlín, 1882 (citado como "Simplicio, In Phys, [pág.].[línea]”).

Everson, S. (1988): “L'explication aristotélicienne du hasard", Revue de la philosophie ancienne 6 (1988), pp. 39-76.

Frede, D. (1992): “Accidental causes in Aristotle”, Synthèse 92 (1992), pp. 39-62.

Freeland, C. (1991): "Accidental Causes and Real Explanations", en L. Judson (ed.) (1991), pp. 49-72. 
Judson, L. (ed.) (1991): Aristotle's Physics. A collection of Essays, Oxford, 1991.

Laplace, P. (1820): Théorie Analytique des Probabilités, en Oeuvres Complètes, Tome Septième, Paris, $1886\left(={ }^{3} 1820\right)$.

Lennox, J. (1982): “Teleology, chance, and Aristotle's Theory of Spontaneous Generation", Journal of the History of Philosophy 20 (1982), pp. 219-238. Ahora en Lennox (2001), pp. 229-249. (Se cita según la paginación de esta última edición, pero por la fecha de edición original del artículo).

- (1984): "Aristotle on Chance", Archiv für Geschichte der Philosophie 66 (1984), pp. 52-60. Ahora en J. Lennox (2001), pp. 250-258. (Se cita según la paginación de esta última edición, pero por la fecha de edición original del artículo).

_ (2001): Aristotle's Philosophy of Biology, Cambridge, 2001.

Loening, R. (1903): Die Zurechnungslehre des Aristoteles, Hildesheim, 1967 (= Jena, 1903).

Long, A., Sedley, D. (1987): The Hellenistic Philosophers, CUP, Cambridge, 1989 (=1987), 2 vols. (citado como Long-Sedley, seguido del número y letra de fragmento).

Madigan, A. (1984): "Metaphysics E 3: a modest proposal", Phronesis 29 (1984), pp. 123-136.

Mansion, A. (1913): Introduction à la Physique Aristotélicienne, Louvain-Paris, 1913. 
MoravcsiK, J. (1991): "What Makes Reality Intelligible? Reflections on Aristotle's Theory of Aitia”, en L. Judson (1991), pp. 31-47.

NAtali, C. (1999): "Problemas de la noción de causa final en Aristóteles”, Anuario Filosófico 32 (1999), pp. 39-57.

Ross (1924) = Aristotle's Metaphysics, a Revised Text with Introduction and Commentary by W. D. Ross, Oxford, 1997 (=1924), 2 vol.

- (1936) $=$ Aristotle's Physics, a revised text with Introduction and Commentary by W. D. Ross, Oxford, 1936.

Schenkl, H. (ed.): Themistii in Aristotelis Physica Paraphrasis, CAG, Vol. V 1-3, Berlín, 1900 (citado como “Temistio, In Phys, [pág.].[línea]”).

Simplicius: On Aristotle's Physics 2, transl. by Barrie Fleet, Cornell University Press, New York, 1997.

Sorabji, R. (1980): Necessity, Cause and Blame: Perspectives on Aristotle's Theory, London, Duckworth, 1980.

Wieland W. (1962): Die aristotelische Physik, Göttingen, 1962.

— (1972): "Zeitliche Kausalstrukturen in der aristotelischen Logik", Archiv für Geschichte der Philosophie 54 (1972), pp. 229-237 (reimpreso en Öffenberger N. y Menne A. [eds.]: Zur modernen Deutung der aristotelischen Logik, Bd. III: Modallogik und Mehrwertigkeit, G. Olms, Hildesheim-Zürich-New York, 1988, pp. 52-60).

ZELLER, E. (1921): Die Philosophie der Griechen in ihrer geschichtlichen Entwicklung, (zweiter Teil, zweite Abteilung) Hildesheim-ZürichNew York, 1990 (Leipzig, ${ }^{4}$ 1921). 
Copyright of Tópicos. Revista de Filosofía is the property of Universidad Panamericana and its content may not be copied or emailed to multiple sites or posted to a listserv without the copyright holder's express written permission. However, users may print, download, or email articles for individual use. 